\title{
Looking for Very Faint X-ray Binaries with INTEGRAL
}

\author{
Melania Del Santo* \\ INAF/IASF-Roma, Italy \\ E-mail: melania.delsantodiasf-roma.inaf.it
}

\section{Lara Sidoli}

INAF/IASF-Milano, Italy

\section{Patrizia Romano}

INAF/IASF-Palermo, Italy

\section{Angela Bazzano}

INAF/IASF-Roma, Italy

\section{Rudy Wijnands}

University of Amsterdam, The Netherlands

\section{Nathalie Degenaar}

University of Amsterdam, The Netherlands

\section{Sandro Mereghetti}

INAF/IASF-Milano, Italy

The improvement of sensitivity of the last generation of X-ray detectors makes a significant number of very faint X-ray binaries with typical luminosities of 1E34-1E36 erg s ${ }^{-1}$ to be detectable. Very faint X-ray binaries, whether transients or persistent, are probably a non-homogeneous class of sources. It is likely that a significant fraction are neutron stars and black holes accreting matter at very low rates from low mass companion. It has been observed that $\sim 1 / 3$ of very faint $X$-ray transients exhibit type-I X-ray bursts, thus they can be identified as neutron star in low mass X-ray binary. In the framework of the INTEGRAL AO6 Key-Programme, we have obtained data rights of a sample of very faint X-ray binaries, being in the Galactic Centre and Galactic Plane. Thanks to the large field of view and its observing programme, INTEGRAL offers the "chance" to catch new type-I X-ray bursts from faint X-ray binaries, as well as to detect faint hard-X ray steady emission at a level of few mCrab. Combination of the INTEGRAL survey with multi-wavelength follow-up (mainly with Swift, and/or XMM-Newton and Chandra), is crucial in unveiling the nature of very faint X-ray binaries and in better refining the global properties of this new class of sources. We present our INTEGRAL and Swift monitoring campaigns, as well as first results of the INTEGRAL AO6 key-programme.

The Extreme sky: Sampling the Universe above $10 \mathrm{keV}$, October 13-17, 2009, Otranto (Lecce) Italy

${ }^{*}$ Poster Presenter 


\section{Very-faint X-ray Transients}

Very Faint X-ray Transients (VFXTs) are a non-homogeneous class of binary systems with outburst peak luminosities in the range $10^{34}-10^{36} \mathrm{erg} \mathrm{s}^{-1}(2-10 \mathrm{keV})$. These luminosities are almost two/three orders of magnitude fainter than the emission typically shown by the bright and faint X-ray transients [20]. However, it is worth to know that the $L_{X}$ boundary as $10^{36} \mathrm{erg} \mathrm{s}^{-1}$ is somewhat arbitrary. While past satellites were mostly able to detect only the brighter transients, as the $\mathrm{X}$-ray detectors became more sensitive (i.e., Chandra and XMM-Newton), fainter transients have been discovered. To date, about 30 members are known (most of them located in the Galactic Centre) and it is likely that most of them are neutron stars (NS) and black holes (BH) accreting matter at very low rates from low-mass companion. A significant fraction $(\sim 1 / 3)$ of the VFXTs exhibited type-I X-ray bursts (Degenaar \& Wijnands 2009 and references therein) and can thus be identified with neutron stars accreting matter from a low-mass (i.e., $M \lesssim 1 \mathrm{M}_{\odot}$ ) companion. However, little is known about VFXTs, in particular concerning the mechanism which drives their very low accretion rate. Some VFXTs display a low (10\%) duty cycle, while others, such as XMMU J174716.1-281048, maintain $\mathrm{L}_{X}=1 \mathrm{E} 34 \mathrm{erg} / \mathrm{s}$ with relatively high $(50 \%)$ duty cycles $[9,8]$. The low outburst luminosities characteristic of VFXTs combined with what is known about their duty cycles, imply that these low-mass X-ray binaries (LMXBs) have very low time-averaged mass accretion rates, which could challenge our understanding of their evolution [17].

A few years ago, another type of accreting neutron stars has been identified, the so-called "burst-only sources" [6]. They are a group of nine objects detected by the BeppoSAX/WFC when exhibiting type I bursts with no persistent emission above the sensitivity limit of the instrument, indicating typical upper limits of $\sim 10^{36} \mathrm{erg} \mathrm{s}^{-1}$. It is possible that these objects are linked to the class of VFXTs [20,4].

\section{INTEGRAL and Swift Monitoring Campaign}

INTEGRAL AO6 observations started on August 2008 and end on December 2009. In the framework of the AO6 key-programme, there are two sky fields containing our selected sample of very faint X-ray binaries (or possible candidates). These 30 sources are all included in a $40^{\circ} \times 40^{\circ}$ Galactic Centre region ( $2 \mathrm{Ms}$ exposure time) and in the two same-size fields of the inner Galactic disc (2 Ms). Based on the INTEGRAL visibility, Galactic Centre was pointed within three periods: Autumn 2008, Spring 2009 and Autumn 2009. As first important result, here we report on the outburst of the "burst-only" source SAX J1753.5-2349 occurred on October 2008 (see Section 4).

Thanks to the combined large IBIS FOV and long exposures, INTEGRAL provides the possibility to detect type-I X-ray bursts or outbursts from very faint transients.

Combining multi-wavelength follow-up allow us the possibility to characterise the sub-luminous accreting NS systems. In particular, we have an on-going monitoring campaign with Swift/XRT with the aim to follow the long-outburst of the burster source XMMU J174716.1-281048 (see Section 3).

\section{Previous results}

The nature of two VFXTs has been unveiled by the INTEGRAL satellite: type-I X-ray bursts 

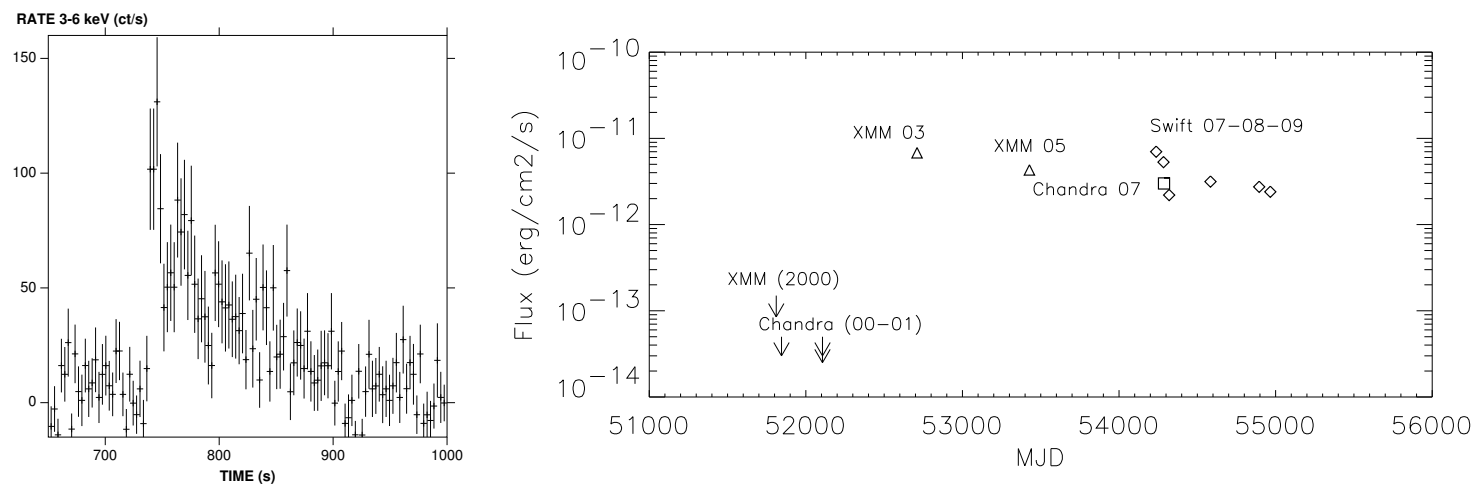

Figure 1: Left: XMMU J174716.1-281048 type-I X-ray burst observed by INTEGRAL/JEM-X in the 3$6 \mathrm{keV}$ band. Right: Long term (2-10 keV) lightcurve of XMMU J174716.1-281048 with XMM-Newton (triangles), Chandra (square) and Swift (diamonds). Upper limits (during quiescence state) are reported with arrows.

with photospheric radius expansion (PRE) were observed from XMMU J174716.1-281048 [9, 2] (see Fig 1, left) and AX J1754.2-2754 [5] during their outbursts at low-luminosity accretion (not observable by INTEGRAL). These observations allowed also to localise the two systems in the Galactic Centre [10]. We note that in the Beppo-SAX era they would have been classified as "burstonly" sources. Based on highly sensitive X-ray satellites (XMM-Newton, Chandra, Swift), it was possible to classify XMMU J174716.1-281048 (Fig. 1, right) and AX J1754.2-2754 as "quasipersistent" X-ray transients because of their prolongated episodes of accretion of the order of a few years $[9,11,1]$.

Current disk instability models do not provide an obvious explanation for outbursts that last several years, displaying a typical $2-10 \mathrm{keV}$ luminosity of a few times $10^{34} \mathrm{erg} \mathrm{s}^{-1}$, as in the case of XMMU J174716.1-281048, still active since its initial discovery in 2003 (Del Santo et al. 2007a; Del Santo et al. 2009). New models are also needed to explain these systems with "quasi-persistent" emission.

\section{High-energy emission from a "burst-only" source}

\subsection{SAX J1753.5-2349}

SAX J1753.5-2349 was discovered in 1996 by Beppo-SAX/Wide Field Camera (WFC) during a single type-I X-ray burst without any steady emission detectable [14]. Thus, it was classified as "burst-only" source [6].

In 2008 October 11, RXTE/PCA, Swift/BAT [18] and INTEGRAL/IBIS [3] detected an outburst from SAX J1753.5-2349 at 10 mCrab flux level. Then, Swift/XRT pointed SAX J1753.5-2349 on October 23 [7], during the decline phase of the outburst (Fig. 2, left). On 2009 March 13, it was re-pointed by Swift and a $3 \sigma$ upper-limit derived [12].

Based on these observations, we have reported for the first time the broad-band spectrum, from soft to hard X-rays, of the persistent emission from a so-called "burst-only" source (see Del Santo et al. 2010). We have studied the 2008 outburst of SAX J1753.5-2349 using all observations 

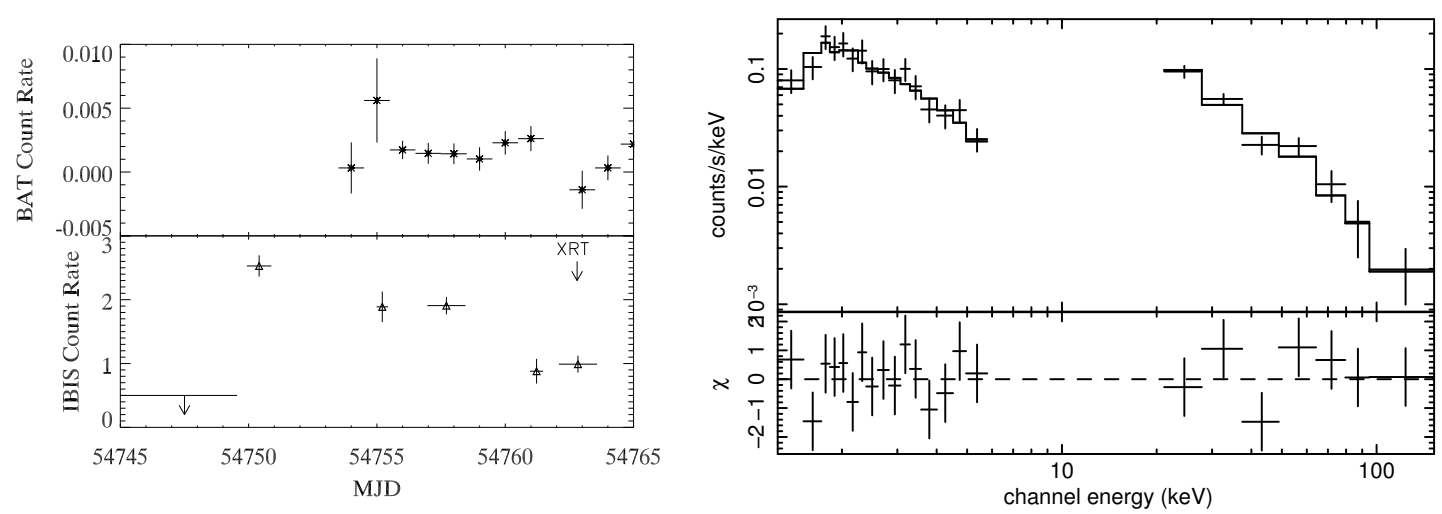

Figure 2: Left: SAX J1753.5-2349 BAT (top) and IBIS/ISGRI (bottom) count rate evolution in the 15-50 $\mathrm{keV}$ and $18-40 \mathrm{keV}$ energy ranges, respectively. The XRT detection time is also shown on the bottom plot. The public BAT light curve starts from 54754 MJD; after MJD=54764 SAX J1753.5-2349 was no longer pointed by INTEGRAL. Right: XRT and IBIS/ISGRI count rate spectra fitted with a simple power-law.

available, including our INTEGRAL observations (P.I. M. Del Santo) in the framework of the AO6 Key-Programme. In the next section we summarise our results.

\subsection{The 2008 outburst}

The outburst from SAX J1753.5-2349 observed with INTEGRAL/IBIS lasted at least 14 days (Fig. 2, left), without any evidence for type-I X-ray bursts, all along the performed INTEGRAL observations of the Galactic Centre region started in 2003. During the outburst, SAX J1753.5-2349 was found in a steady low/hard spectral state empirically described by a simple power law with $\Gamma \sim 2$ (Fig. 2, right). Physically, the $1-200 \mathrm{keV}$ spectrum is most likely produced by the upscattering of soft seed photons by a hot optically thin electron plasma (i.e. Barret et al. 2000 and references therein). By fitting the spectrum with comptt the electron temperature is not constrained, while a lower limit of $\sim 24 \mathrm{keV}$ (at 90\%) can be inferred [13]. Even though, a black-body emission from the neutron star surface is also expected to be observed in the low/hard states of bursters, in SAX J1753.5-2349 it is not detected, possibly because of the high absorption observed $\left(\mathrm{N}_{H} \sim 2 \times 10^{22} \mathrm{~cm}^{-2}\right)$.

We noted that SAX J1753.5-2349 displays very-faint outbursts $\left(L_{X}<10^{36} \mathrm{erg} \mathrm{s}^{-1}\right.$, in 1996 based on Beppo-SAX/WFC observations), as well as outbursts with luminosities in the range 10 $0^{36-37}$ $\mathrm{erg} \mathrm{s}^{-1}$ (in 2008), which are classified as Faint X-ray Transients (FXT; Wijnands et al. 2006). Although it is probably an hybrid system (displaying both faint and very faint outbursts) the observed luminosities confirm the hypothesis that the so-called "burst-only" sources belong to the class of the subluminous neutron star X-ray binaries. We also estimated the duty cycle $\left(t_{\mathrm{ob}} / t_{\mathrm{rec}}\right)$ of the system which ranges from a minimum of $0.3 \%$ to a maximum of $9 \%$ and into a long-term time-averaged accretion rate $\left(\left\langle\dot{M}_{\text {long }}\right\rangle=\left\langle\dot{M}_{\mathrm{ob}}\right\rangle \times t_{\mathrm{ob}} / t_{\mathrm{rec}}\right)$ ranging from $2.2 \times 10^{-12}$ to $6.0 \times 10^{-11} \mathrm{M}_{\odot} \mathrm{yr}^{-1}[13]$.

Following in't Zand et al. (2007), we have estimated the hardness ratio 40-100/20-40 keV within each INTEGRAL revolutions. We find a value consistent with 1 which confirms the hard nature of the system. This is also consistent with the low mass accretion rate inferred (see also Paizis et al. 2006), which confirms that SAX J1753.5-2349 is not a fake faint system and there would be no reason to assume that the system is obscured to explain the low $\dot{M}$. Moreover, King 
(2000) argued that the faint low-mass X-ray transients are mainly neutron star X-ray binaries in very compact binaries with orbital periods lower than 80 min. We suggest that the SAX J1753.5-2349 system is a good candidate to harbor an accreting neutron star in a very compact system.

A sample of the low-luminosity transient LMXBs (including SAX J1753.5-2349) displays very different behaviours in terms of peak luminosity, outburst duration and recurrence time from year to year. This behaviour is one of the still open issues in the VFXTs research field: in particular, it is not well understood whether these variations should be interpreted as being due to changes in the mass-transfer rate or as results of instabilities in a cool accretion disk.

\section{Acknowledgments}

Data analysis is supported by the Italian Space Agency (ASI), via contract ASI/INTEGRAL I/008/07/0. MDS thanks Memmo Federici for the INTEGRAL data archival support at IASF-Roma. We thank N. Gehrels for accepting our Swift ToO proposals and Swift team. This proceeding is based on observations with INTEGRAL, an ESA project with instruments and science data centre funded by ESA member states (especially the PI countries: Denmark, France, Germany, Italy, Switzerland, Spain), Czech Republic and Poland, and with participation of Russia and the USA.

\section{References}

[1] Bassa C., et al., 2008, ATel \#1575

[2] Brandt, S., et al. 2006, ATel 970

[3] Cadolle Bel M., et al., 2008, ATel \#1910

[4] Campana S., 2009, ApJ, 699, 1144

[5] Chelovekov I. V., \& Grebenev S. A., 2007, ATel \#1094

[6] Cornelisse R., et al., 2004, Nucl. Phys., 132, 518

[7] Degenaar N., \& Wijnands R., 2008, ATel \#1809

[8] Degenaar N., \& Wijnands R., 2009, A\&A, 495, 547

[9] Del Santo M., et al., 2007a, A\&A, 468, L17

[10] Del Santo M., et al., 2007b, ATel \#1207

[11] Del Santo M., et al., 2008, AIPC, 1010, 162

[12] Del Santo M., et al., 2009, ATel \#1975

[13] Del Santo M., et al., 2010, MNRAS, in press. (astro-ph/1001.5410)

[14] in't Zand J. J. M., et al., 1999, Nucl. Phys, 69, 228

[15] in't Zand J. J. M., Jonker P. G., Markwardt C. B., 2005, A\&A, 465, 953

[16] King, A. R., 2000, MNRAS, 315, L33

[17] King A. R.\& Wijnands R., 2006, MNRAS, 366, L31

[18] Markwardt C. B., Krimm H. A., Swank J. H., 2008, ATel \#1799

[19] Paizis A., et al. 2006, A\&A, 459, 187

[20] Wijnands R., et al., 2006, A\&A, 449, 1117 